\title{
Renal Function and Long-Term Decline in Cognitive Function: The Baltimore Longitudinal Study of Aging
}

\author{
Stephen L. Seliger ${ }^{a}$ Carrington R. Wendell ${ }^{c} d$ Shari R. Waldstein ${ }^{b, c, e}$ \\ Luigi Ferrucci $^{d}$ Alan B. Zonderman ${ }^{d}$ \\ ${ }^{a}$ Division of Nephrology and ${ }^{b}$ Division of Gerontology and Geriatric Medicine, Department of Medicine, University \\ of Maryland School of Medicine, Baltimore, Md., ' Department of Psychology, University of Maryland, Baltimore \\ County, Md., dintramural Research Program, National Institute of Aging, NIH, and e Geriatric Research Education and \\ Clinical Center, Baltimore VA Medical Center, Baltimore, Md., USA
}

\section{Key Words \\ Kidney function $\cdot$ Creatinine $\cdot$ Estimated glomerular filtration rate $\cdot$ Cognitive function $\cdot$ Neuropsychology . Longitudinal trajectories}

\begin{abstract}
Background: Renal disease has been associated with greater risk of dementia and greater cognitive impairment. However, the relationship of lower renal function with long-term decline in specific domains of cognitive function remains unclear among community-dwelling, non-demented individuals. Methods: Stroke- and dementia-free participants ( $\mathrm{n}=$ 2,116 ) were enrolled in the Baltimore Longitudinal Study of Aging, a community-based, prospective, longitudinal study. Renal function was estimated by the inverse of serum creatinine adjusted for age, sex and race and (in sensitivity analyses) estimated glomerular filtration rate (eGFR) using the MDRD formula. Outcome measures were changes in scores on 6 cognitive tests encompassing a range of cognitive functions, measured at 2-year intervals. Mixed-effects regression models examined the longitudinal relations of renal function with cognitive functions after adjusting for demographics, comorbidity and other potential confounders. Results: Mean age at initial testing was 53.9 years (SD 17.1), and 94
\end{abstract}

participants (4.4\%) had an eGFR $<60 \mathrm{ml} / \mathrm{min} / 1.73 \mathrm{~m}^{2}$ and $18.5 \%$ had at least one comorbidity. With increasing age, longitudinal increases in creatinine concentrations were associated with more rapid decline in performance on several cognitive measures, including the learning slope of the California Verbal Learning Test, a test of verbal learning $(p<$ 0.01 ), and the Benton Visual Retention Test, a test of visual memory $(p<0.01)$. Associations were similar for changes in eGFR $R_{M D R D}$, which was also associated with the rate of decline in verbal memory. Conclusion: In a community-based adult population, declines in renal function independently associated with greater long-term declines in visual memory and verbal memory and learning.

(c) 2015 National Institutes of Health (NIH) Published by S. Karger AG, Basel

\section{Introduction}

Cognitive function declines with age, but the rate of decline varies greatly among individuals. The presence of cardiovascular risk factors such as hypertension, diabetes and dyslipidemia may explain some of the variability in age-related decline and in particular, may accelerate the rate of decline or the risk of incident cognitive impairment

\begin{tabular}{ll}
\hline KARGER 125/s & $\begin{array}{l}\text { ○ 2015 National Institutes of Health (NIH) } \\
\text { Published by S. Karger AG, Basel } \\
0250-8095 / 15 / 0415-0305 \$ 39.50 / 0\end{array}$ \\
$\begin{array}{l}\text { E-Mail karger@karger.com } \\
\text { www.karger.com/ajn }\end{array}$ &
\end{tabular}


$[1,2]$. Chronic kidney disease (CKD) is a highly prevalent condition [3] that increases with age and is strongly related to cardiovascular risk [4], including risk of cerebrovascular diseases [5]. A major role for CKD in accelerating cognitive decline with aging is therefore plausible, although prior studies testing this hypothesis have been limited.

Among patients with end-stage renal disease on maintenance dialysis, several cross-sectional studies have reported a high prevalence of cognitive impairment and lower average cognitive function when compared to controls with preserved renal function $[6,7]$. In study samples from the general population and from patients with selected comorbidities, lower average neurocognition was also observed in association with mild-moderate decrements of renal function, suggesting that impairments are not limited to those with severe azotemia [8-10]. However, prior studies were limited by either a cross-sectional design $[8,9,11]$ or by the use of global mental status measures (e.g. the Mini Mental State Examination (MMSE) $[10,12]$, which has limited sensitivity and specificity in detecting age-related changes in different cognitive functions with aging.

The primary aim of this study was to determine the association of changes in renal function with longitudinal changes in specific domains of cognitive function among community-dwelling, stroke- and dementia-free adults, using repeated assessments of multiple cognitive functions performed in the Baltimore Longitudinal Study of Aging (BLSA). We hypothesized that declining renal function would be associated with a more rapid decline in cognitive function.

\section{Materials and Methods}

\section{Study Sample}

The BLSA is a prospective cohort study of community-dwelling participants conducted by the National Institute on Aging's Intramural Research Program since 1958. Approximately every 2 years, participants visit the National Institute on Aging in Baltimore, Maryland, for medical, psychological and cognitive testing. Beginning in 1986, a more extensive neuropsychological test battery was administered at each visit to participants who were $\geq 60$ years. The majority of tests for which data were analyzed in this study were therefore available only after 1986. The BLSA uses continuous enrollment procedures; thus, the years of enrollment and number of total visits differ among participants. In addition, for any given participant, the number of measures for each cognitive test may differ, depending on the time when a patient entered the study and in what year a given test was introduced to the test battery. For the purposes of the present analysis, participants with prior or incident stroke or a prior or incident diagnosis of dementia were excluded.
Institutional Review Board approval was obtained from the Johns Hopkins Bayview Medical Center prior to 2002 and the MedStar Research Institute after 2002. All participants provided written informed consent. Data analyses were approved by the Institutional Review Board of the University of Maryland, Baltimore County.

\section{Neuropsychological Tests}

At each BLSA visit, a neuropsychological test battery was administered by trained psychometricians. The Digits Forward and Digits Backward portions of the Wechsler Adult Intelligence ScaleRevised assessed the attention and concentration/working memory. Verbal learning and memory were assessed using the California Verbal Learning Test (CVLT), with derived scores including short and long free recall and the learning slope. Visual memory was assessed using the Benton Visual Retention Test (BVRT). The trailmaking test parts A and B assessed attention, visuomotor scanning, perceptuomotor speed and mental flexibility (an executive function). Other tests included Letter and Category Fluency (phonemic and semantic association fluency, respectively, and executive function) and the Boston Naming Test (confrontation naming). The MMSE was administered as a screening test for general cognition; MMSE scores were not considered an outcome variable in this analysis due to the narrow range of scores observed.

\section{Primary Predictor Variable}

The primary measure of renal function was the inverse of serum creatinine (1/SCr). The decision to use $1 / \mathrm{SCr}$ rather formuladerived estimates of GFR was motivated by the use of mixed-regression models to estimate associations with change in cognitive function (see 'Statistical Analysis', below). The primary measures of association in these mixed-effects models are the coefficients for the interaction between age and renal function. Therefore, including MDRD- or CKD-Epi-estimated glomerular filtration rate (eGFR) - which itself involves an interaction between age and 1/ $\mathrm{SCr}$ - as the primary measure of renal function would result in a model with significant challenges to interpretation. However, by accounting for factors other than GFR which influence creatinine levels - age, gender, race, body mass - as independent covariates in the mixed model, the model coefficients for age* $1 / \mathrm{Scr}$ can be reasonably interpreted as primarily representing the association of renal filtration function with changes in cognitive function. However, given the widespread use of eGFR in both clinical care and epidemiologic study of renal disease, we performed secondary analyses by replacing $1 / \mathrm{SCr}$ with eGFR using the 4 -variable MDRD equation as the primary predictor variable.

\section{Covariates}

Age was scaled per decade, and education was measured in years. Mean arterial pressure was calculated as $(2 * \mathrm{DBP}+\mathrm{SBP}) / 3$ from seated blood pressure measures at each study visit. Body mass index was calculated as the ratio of weight (in kilograms) to height (in meters) squared, both measured with a clinical calibrated scale. Medication use including antihypertensives, non-steroidal antiinflammatory drugs (NSAIDs) and aspirin and was coded as yes/ no at each visit. Laboratory measurements were performed at each study visit at the clinical laboratories of the National Institute on Aging. Laboratory data abstracted for the present analysis included hemoglobin and creatinine. Because the frequency of specific individual comorbidities was low in this relatively healthy cohort, we used a comorbidity index score representing the number of dif- 
ferent comorbid conditions (identified by the study physician at each visit from medical history) including coronary artery disease, myocardial infarction, congestive heart failure, peripheral arterial disease, transient ischemic attack and diabetes mellitus. Cigarette smoking was characterized as current versus former or never.

\section{Statistical Analysis}

All statistical analyses were performed using SAS, version 9.2 (Cary, N.C., USA). Mixed-effects linear regression models were developed to examine the longitudinal relations between renal and cognitive functions. Mixed-effects models are the preferred method for analyzing data in which repeated outcome measures are assessed at non-uniform intervals among individuals (i.e. unbalanced data). They allow for both fixed and random terms, accounting for correlation of repeated measures within individuals and are appropriate in the setting of variable lengths of follow-up among individual participants. Both renal and cognitive functions were measured at each BLSA visit; analyses thus examine coincident trajectories of renal and cognitive functions over time.

In order to maximize the information associated with each neuropsychological test, separate mixed models were developed for each test. Adjustment covariates were the same for each neurocognitive outcome and were selected apriori, based on factors which could plausibly relate to cognitive decline, based on prior studies of the BLSA [13], and/or were plausibly related to renal function and SCr levels and included age, sex, education, mean arterial pressure, hemoglobin, smoking, comorbidity score, NSAID use and use of aspirin. All covariates other than sex were treated as time varying. In sensitivity analyses, additional adjustment was made for race, body mass and the use of antihypertensive medications.

Age was also modeled as a random term to index time [14]. The practice of using age as a metric for time is recommended by McArdle and Bell [15] for instances in which a study lacks a natural baseline due to randomness of time of study entry, which is the case in the BLSA. The distribution of participants over time is regarded as a sample of performance opportunities across ages. With sufficient numbers of participants over a sufficiently broad range of ages, we can examine the overall differences in rates of change in time-varying effects. As a result, individuals with only a single measurement are able to contribute information to the analyses.

Models included both main and interactive, longitudinal terms of interest. Significant main effects of renal function indicate that the cognitive outcome (collapsed across all available testing sessions) is associated with overall differences in renal function, regardless of longitudinal change. Longitudinal change in the cognitive outcome associated with renal function is indicated by the interaction term of renal function and age. The latter reflects age-related differences in cognitive decline as a function of prospectively assessed changes in renal function. $p$ values $<0.05$ were considered statistically significant.

\section{Results}

\section{Study Participants}

After exclusion of participants with stroke and/or dementia $(n=172)$, a total of 2,116 participants received at least one cognitive test. The BVRT was the test for which
Table 1. Characteristics of study sample at first BLSA visit

\begin{tabular}{|c|c|c|}
\hline Variable & $\begin{array}{l}\text { Mean or } \\
\text { percentage }\end{array}$ & $\mathrm{SD}$ \\
\hline Age, years & 53.9 & 17.1 \\
\hline \multicolumn{3}{|l|}{ Age, $\%$} \\
\hline $17-29$ & 9.8 & \\
\hline $30-39$ & 14.5 & \\
\hline $40-49$ & 14.5 & \\
\hline $50-59$ & 20.8 & \\
\hline $60-69$ & 17.6 & \\
\hline $70-79$ & 16.4 & \\
\hline $80-94$ & 6.1 & \\
\hline Education, years & 16.7 & 2.8 \\
\hline Sex, male, \% & 61.6 & \\
\hline Race, white, \% & 85.5 & \\
\hline $\mathrm{MAP}, \mathrm{mm} \mathrm{Hg}$ & 95.7 & 13.2 \\
\hline Hemoglobin, g/dl & 14.5 & 1.4 \\
\hline Smokers, \% & 20.0 & \\
\hline NSAID use, $\%$ & 13.9 & \\
\hline Aspirin use, \% & 11.5 & \\
\hline Antihypertensive medications, $\%$ & 16.4 & \\
\hline Comorbidity index $>0, \%$ & 18.5 & \\
\hline Diabetes mellitus, \% & 4.9 & \\
\hline Serum creatinine, $\mathrm{mg} / \mathrm{dl}$ & 0.81 & 0.22 \\
\hline $\mathrm{eGFR}, \mathrm{ml} / \mathrm{min} / 1.73 \mathrm{~m}^{2}$ & 103.2 & 26.6 \\
\hline $\mathrm{eGFR}<60 \mathrm{ml} / \mathrm{min} / 1.73 \mathrm{~m}^{2}, \%$ & 4.4 & \\
\hline MMSE, total score & 28.3 & 1.8 \\
\hline
\end{tabular}

MAP $=$ Mean arterial pressure. eGFR was calculated using the 4-variable MDRD equation of Levey et al.

data were provided by the largest participant sample. The characteristics of the participants at the first BVRT administration are listed in table 1 . A total of 94 participants $(4.4 \%)$ had an eGFR $<60 \mathrm{ml} / \mathrm{min} / 1.73 \mathrm{~m}^{2}$. The number of participants contributing data for each cognitive test and the number of test administrations per individual are shown in supplementary table 1 online (for all online suppl. material, see www.karger.com/doi/ $10.1159 / 000430922)$. Each test was administered over a median of $4-5$ visits per individual over a mean of 7.7 years.

Association of 1/SCr and Cognitive Functional Decline A significant age at $1 / \mathrm{SCr}$ interaction was observed for the CVLT learning slope $(b=0.126, p=0.008)$. Figure 1a shows the model-based predicted CVLT score as a function of time and SCr level; with increasing levels of creatinine, the rate of decline of test performance is more rapid. A significant longitudinal age at $1 / \mathrm{SCr}$ interaction 


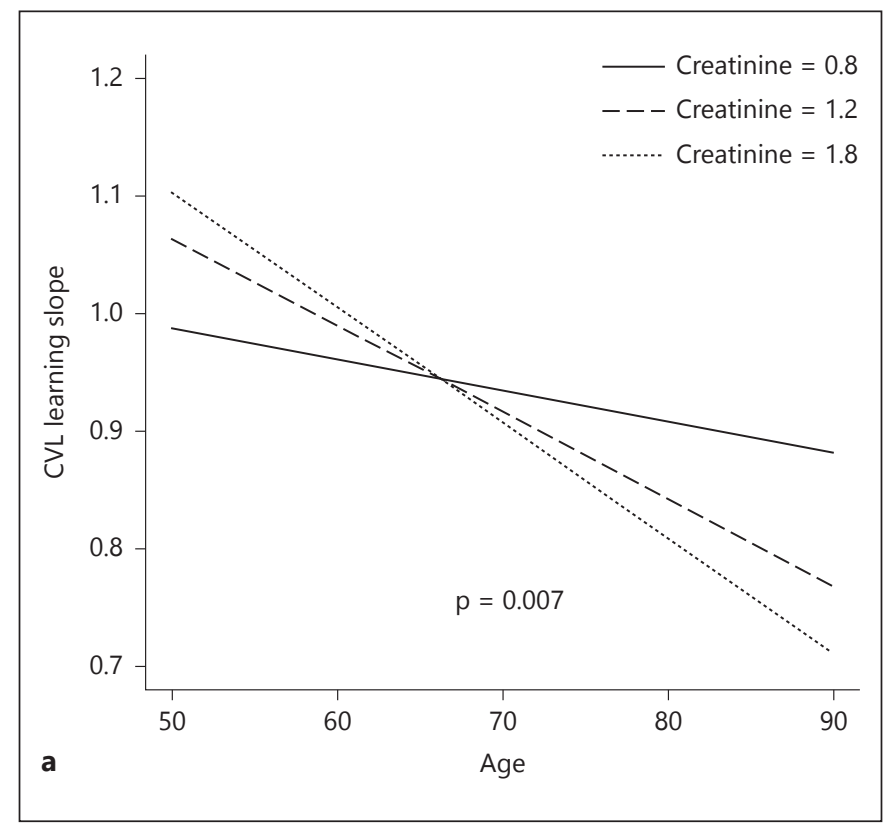

Fig. 1. a Rate of change in verbal learning (CVLT learning slope) by SCr concentration. $\mathbf{b}$ Change in visual memory of men (\# errors on BVRT) by SCr concentration. c Change in visual memory of women (\# errors on BVRT) by SCr concentration.

was also observed for the BVRT; however, the association differed significantly between men and women (test for interaction by sex, $\mathrm{p}=0.002$ ). Figure $1 \mathrm{~b}$ and $\mathrm{c}$ show the model-based estimated scores for the BVRT as a function of age and SCr, for both men (b) and women (c). The rate of decline in performance on the BVRT was greater with increasing levels of creatinine, but the association was more pronounced among men than among women. No significant independent associations of $1 / \mathrm{SCr}$ with rate of
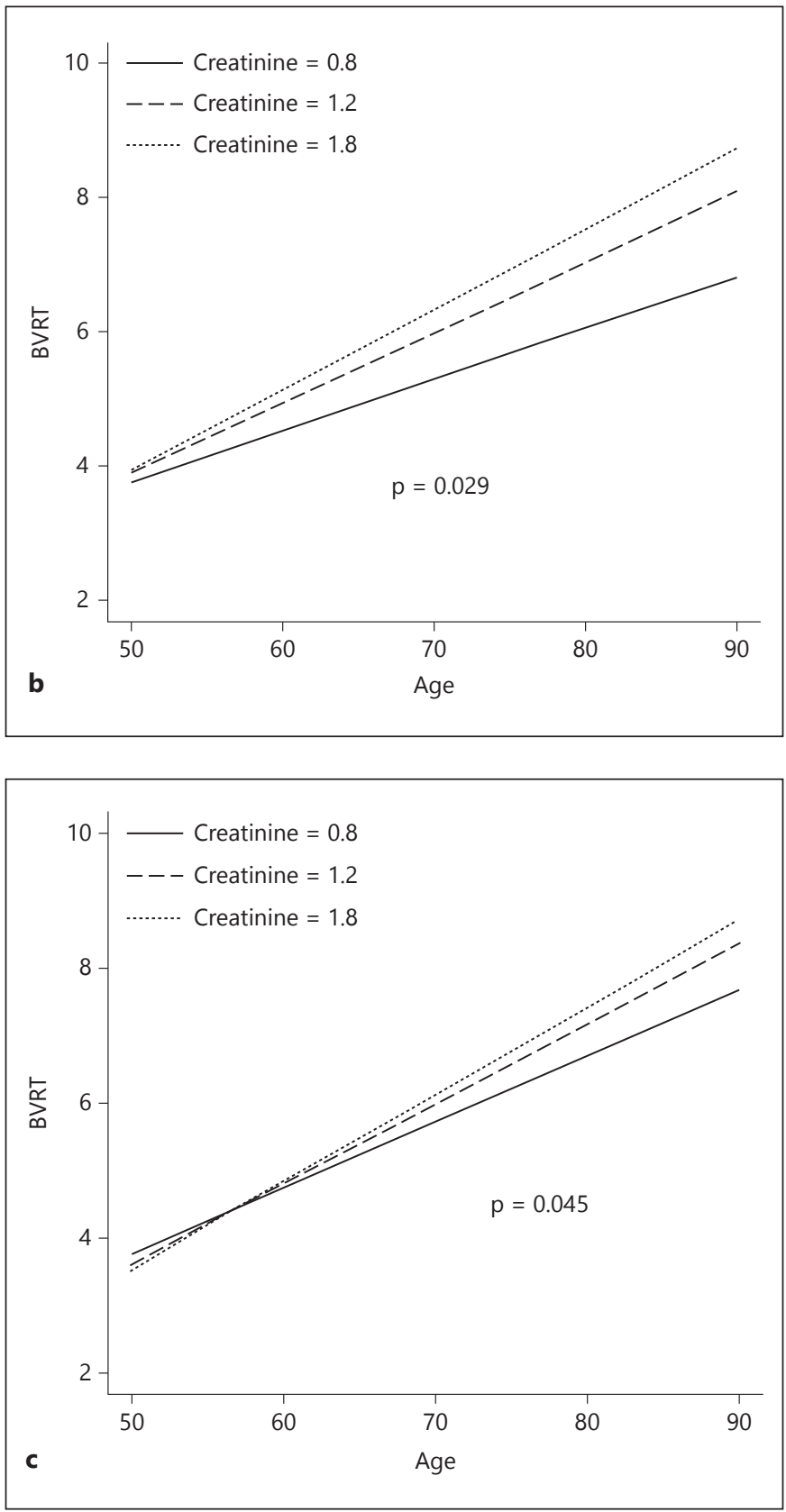

change in the other cognitive tests scores were observed, although performances on most tests were observed to decline over time (table 2).

No significant departure from linearity was observed for the longitudinal associations of $1 / \mathrm{SCr}$ with cognitive function. Additional adjustment for race, body mass, and use of antihypertensive medications (the latter two as time-varying covariates) did not materially change the results. Specifically, there remained a significant $1 / \mathrm{SCr}$ age 
Table 2. Coefficients from mixed-effects regression models relating change in $1 / \mathrm{Scr}$ with change in cognitive function

\begin{tabular}{|c|c|c|c|c|}
\hline & Test score & Age & Age $^{*} 1 / \mathrm{SCr}^{\mathrm{a}}$ & Age $^{*} \operatorname{sex}^{*} 1 / \mathrm{Scr}^{\mathrm{b}}$ \\
\hline BVRT & \# errors & $1.611^{* *}$ & $-0.510^{* *}$ & $-0.165^{* *}$ \\
\hline Boston naming test & \# correct & -1.294 & 0.488 & \\
\hline CVLT learning slope & mean \# of new words per trial & $-0.191^{* *}$ & $0.126^{* *}$ & \\
\hline CVLT free recall, short delay & \# correct & $-1.257^{* *}$ & 0.337 & \\
\hline CVLT free recall, long delay & \# correct & $-1.335^{* *}$ & 0.486 & \\
\hline Digit span test, forward & \# correct & -0.251 & -0.020 & \\
\hline Digit span test, backward & \# correct & -0.247 & -0.033 & \\
\hline Category fluency & \# correct & $-1.768^{* *}$ & 0.256 & \\
\hline Letter fluency & \# correct & -0.667 & 0.119 & \\
\hline Trail-making test, part A & time to completion & $6.165^{*}$ & 1.133 & \\
\hline Trail-making test, part B & time to completion & $19.48^{*}$ & 6.906 & \\
\hline
\end{tabular}

interaction for the CVLT learning slope $(\mathrm{p}<0.05)$ and for the BVRT $(\mathrm{p}<0.01)$, with a greater association of higher creatinine with age-related decline in the BVRT among men than among women $\left(\mathrm{p}<0.001\right.$ for $1 / \mathrm{Scr}^{*}$ age*sex interaction). Likewise, additional adjustment for the assay method for SCr (as a time-varying covariate) did not change the results (online suppl. table 2). In an additional sensitivity analysis, we examined whether associations differed after excluding those with prevalent cardiovascular disease and eGFR $<60 \mathrm{ml} / \mathrm{min} / 1.73 \mathrm{~m}^{2}$ at baseline. Results were largely unchanged: a significant age*1/SCr interaction was still observed for the CVLT slope (online suppl. table 3) with greater age-related decline with higher SCr levels, and a significant longitudinal age*1/SCr interaction was observed for the BVRT, with greater agerelated decline with higher SCr levels and more pronounced associations among men than among women. There was a significant age* $1 / \mathrm{SCr}^{*}$ sex interaction for performance on the trail-making test part B; however, in neither men nor women was the decline in test performance related to SCr, and the interaction was driven largely by 4 influential outlying values.

\section{Secondary Analysis: Association of eGFR and}

Longitudinal Cognitive Function Changes

Significant associations $(\mathrm{p}<0.01)$ were also observed between eGFR and change in performance on the BVRT and CVLT learning slope, with the direction of the regression coefficients indicating slower decline with higher
eGFR (table 3). Significant $(\mathrm{p}<0.05)$ associations were also observed between higher eGFR and slower declines in performance on the CVLT short- and long-delay free recall and tests of verbal memory. No significant interactions were observed for changes in performance on Digit Span, Letter Frequency or Category frequency tests. For changes on the trail-making test parts $\mathrm{A}$ and $\mathrm{B}$, the relationship of eGFR with decline in performance differed significantly $(p<0.01)$ by gender, but among neither men nor women was the relationship significant.

\section{Discussion}

Among community-dwelling, stroke- and dementiafree adults followed longitudinally, declining renal filtration was associated with a more rapid decline in the cognitive functions of verbal learning/memory and visual memory, after adjustment for potential demographic and some potentially confounding factors. In contrast, changes in renal function were not associated with a more rapid decline on tests of naming, executive function, language fluency or psychomotor speed. These findings are notable as the study sample was relatively healthy, with only $19 \%$ having diabetes or cardiovascular disease. Furthermore, differences in rates of cognitive decline were observed over a relatively modest range of decreased renal function, and among participants without evidence of global cognitive impairment, 


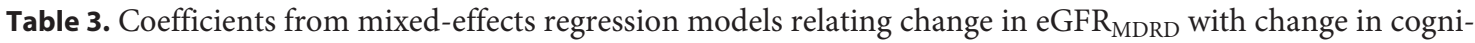
tive function

\begin{tabular}{|c|c|c|c|c|}
\hline & Test score & Age & Age* $^{*} / \mathrm{eGFR}^{\mathrm{a}}$ & Age*sex*eGFR $^{b}$ \\
\hline BVRT & \# errors & $1.664^{* *}$ & $-0.008^{* *}$ & \\
\hline Boston naming test & \# correct & $-1.496^{*}$ & 0.011 & \\
\hline CVLT learning slope & mean \# of new words per trial & $-0.171^{* *}$ & $0.002^{* *}$ & \\
\hline CVLT free recall, short delay & \# correct & $-1.338^{* *}$ & $0.005^{*}$ & \\
\hline CVLT free recall, long delay & \# correct & $-1.342^{* *}$ & $0.006^{*}$ & \\
\hline Digit span test, forward & \# correct & -0.300 & 0.0001 & \\
\hline Digit span test, backward & \# correct & -0.240 & -0.001 & \\
\hline Category fluency & \# correct & $-1.700^{* *}$ & 0.003 & \\
\hline Letter fluency & \# correct & -0.650 & 0.005 & \\
\hline Trail-making test, part A & time to completion & $7.107^{* *}$ & 0.005 & $-0.037^{*}$ \\
\hline Trail-making test, part B & time to completion & $20.436^{*}$ & 0.118 & $-0.168^{* *}$ \\
\hline \multicolumn{5}{|c|}{$\begin{array}{l}* \mathrm{p}<0.05 ;{ }^{* *} \mathrm{p}<0.01 \text {. } \\
\text { a Test of the association of eGFR with change in score on cognitive test. } \\
\mathrm{b} \text { Test of the interaction between eGFR and sex on change in cognitive test score. Blank spaces indicate that } \\
\text { significant }(\mathrm{p}<0.05) \text { interaction was detected. A negative coefficient indicates the relationship between the } \\
\text { rameters. }\end{array}$} \\
\hline
\end{tabular}

as evidenced by excluding participants with dementia diagnoses.

Results of our investigation are not directly comparable to prior studies of CKD and cognition given the differences in the specific cognitive tests used as well as differences between longitudinal and cross-sectional designs. Nevertheless, among prior studies, lower function in the domains of verbal and visual memory and learning has also been reported in association with end-stage renal disease [6], non-dialysis CKD [16] and lower renal function [11]. The current study suggests that not only do individuals with renal impairment have lower function in verbal and/or visual memory, but also that they experience a faster rate of decline in these functions with aging. However, we did not find a relationship between declining renal function and more rapid decline on tests of psychomotor speed and complex attention, in contrast to some prior studies which found associations of renal impairment and lower performance in these functional domains. Although most of these studies were cross-sectional, a longitudinal analysis of older adults identified lower renal function as a predictor of more rapid decline in the performance of Digit Symbol Substitution Test, a measure of complex attention and set-shifting [17]. Other functional domains including memory and learning were not assessed in that study.

The factors which explain the associations between reduced renal function and more rapid age-related decline in cognitive functions remain uncertain, although a number of mechanisms have been proposed. For example, patients with renal impairment have associated systemic and cerebral vascular changes. Systemic changes include reduced vascular compliance and greater aortic stiffness, which have been linked to worse cognitive performance in older adults. Within the brain, even modestly lower renal function has been associated with a greater burden of white matter hyperintensities on cranial imaging [18, 19], linked to cognitive impairment. In support of this hypothesis, we have previously reported that moderate renal impairment was associated with a greater long-term risk of incident vascular-type dementia in older adults, even after accounting for comorbidity [20]. Other potential factors linking renal impairment and disease with cognitive dysfunction include microinflammation, oxidative stress, hyperhomocysteinemia and altered nitric oxide metabolism. However, the causal relationships among renal function, cognitive function and these physiological factors remain uncertain.

The mechanisms underlying the sex difference in relation of renal decline to BVRT decline remain unclear. Visual memory performance is heavily reliant on medial temporal lobe function, and animal research suggests that age-related hippocampal atrophy is more pronounced among men than among women [21]. However, the absence of sex differences between renal function and CVLT performance suggests that other factors are involved, giv- 
en that verbal memory performance is also reflective of hippocampal function.

Limitations of the study include the use of SCr as a renal function marker, which can result in misclassification of true GFR due to interindividual differences in creatinine generation and tubular secretion. However, results were similar when eGFR was used as an alternative renal function marker. The BLSA is a sample of convenience and does not represent a true community- or population-based study sample. Participants were well educated as compared to the general population and were recruited from one geographic location; therefore, the results may not be generalizable to other populations. We cannot exclude the possibility of a type I (false-positive) error in the setting of examination of associations with multiple cognitive test outcomes. However, the similarity of results when either $1 / \mathrm{SCr}$ or eGFR was used as a renal function marker suggests that the observed associations with visual and verbal memory are not due to chance alone. Finally, this study sample did not have measurements of albuminuria, which has also been linked to cognitive dysfunction independent of renal filtration function $[22,23]$.

Strengths of the study include the longitudinal study design, the more extensive cognitive test battery administered and the evaluation of multiple potential confounders. Simultaneous examination of decline in kidney function in relation to decline in cognitive function rep- resents a unique contribution to the literature and parallels the recent findings involving the decline of kidney function and prospective cardiovascular risk [24].

\section{Conclusions}

In conclusion, among a community-dwelling adult population, modest decline in renal function is associated with a greater decline in verbal memory/learning and visual memory with age, after accounting for differences in demographics and comorbidity, whereas no associations were observed with decline in other cognitive domains. These findings are notable in the context of a relatively healthy sample (i.e. low comorbidity), across a modest range of decreased renal function and without cognitive impairment. Future investigation is needed to determine the factors that explain these associations.

\section{Acknowledgment}

The National Institute on Aging Intramural Research Program of the National Institutes of Health supported this research.

\section{Disclosure Statement}

The authors have no conflicts of interest to disclose.

\section{References}

1 Waldstein SR, Elias MF (eds): Neuropsychology of Cardiovascular Disease. Mahwah, NJ, Lawrence Erlbaum Associates, 2001.

2 Waldstein SR, Wendell CR, Hosey MM, Seliger SL, Katzel LI: Cardiovascular disease and neurocognitive function; in Armstrong C, Morrow LA (eds): Handbook of Medical Neuropsychology: Applications of Cognitive Neuroscience. New York, Springer, 2010, pp 69-99.

3 Coresh J, Selvin E, Stevens LA, Manzi J, Kusek JW, Eggers P, Van Lente F, Levey AS: Prevalence of chronic kidney disease in the United States. JAMA 2007;298:2038-2047.

4 Go AS, Chertow GM, Fan D, McCulloch CE, Hsu CY: Chronic kidney disease and the risks of death, cardiovascular events, and hospitalization. N Engl J Med 2004;351: 1296-1305.

5 Seliger SL, Longstreth WT Jr: Lessons about brain vascular disease from another pulsating organ, the kidney. Stroke 2008; 39:5-6.
6 Murray AM, Tupper DE, Knopman DS, Gilbertson DT, Pederson SL, Li S, Smith GE, Hochhalter AK, Collins AJ, Kane RL: Cognitive impairment in hemodialysis patients is common. Neurology 2006;67:216-223.

7 Sehgal AR, Grey SF, DeOreo PB, Whitehouse PJ: Prevalence, recognition, and implications of mental impairment among hemodialysis patients. Am J Kidney Dis 1997; 30:41-49.

8 Hailpern SM, Melamed ML, Cohen HW, Hostetter TH: Moderate chronic kidney disease and cognitive function in adults 20 to 59 years of age: third national health and nutrition examination survey (NHANES III). J Am Soc Nephrol 2007;18:2205-2213.

9 Kurella M, Yaffe K, Shlipak MG, Wenger NK, Chertow GM: Chronic kidney disease and cognitive impairment in menopausal women. Am J Kidney Dis 2005;45:66-76.

10 Kurella Tamura M, Wadley V, Yaffe K, McClure LA, Howard G, Go R, Allman RM, Warnock DG, McClellan W: Kidney function and cognitive impairment in US adults: the reasons for geographic and racial differences in stroke (REGARDS) study. Am J Kidney Dis 2008;52:227-234.

11 Elias MF, Elias PK, Seliger SL, Narsipur SS, Dore GA, Robbins MA: Chronic kidney disease, creatinine and cognitive functioning. Nephrol Dial Transplant 2009;24:2446-2452.

12 Kurella M, Chertow GM, Fried LF, Cummings SR, Harris T, Simonsick E, Satterfield S, Ayonayon H, Yaffe K: Chronic kidney disease and cognitive impairment in the elderly: the health, aging, and body composition study. J Am Soc Nephrol 2005; 16:21272133.

13 Wendell CR, Zonderman AB, Metter EJ, Najjar SS, Waldstein SR: Carotid intimal medial thickness predicts cognitive decline among adults without clinical vascular disease. Stroke 2009;40:3180-3185.

14 Waldstein SR, Giggey PP, Thayer JF, Zonderman AB: Nonlinear relations of blood pressure to cognitive function: the Baltimore longitudinal study of aging. Hypertension 2005; 45:374-379. 
15 McArdle JJ, Bell RQ: An introduction to latent growth models for developmental data analysis; in Little TD, Schnabel KU, Baumert J (eds): Modeling Longitudinal and Multilevel Data: Practical Issues, Applied Approaches, and Specific Examples. Mahwah, NJ, Lawrence Erlbaum Associates, 2000.

16 Thornton WL, Deria S, Gelb S, Shapiro RJ, Hill A: Neuropsychological mediators of the links among age, chronic illness, and everyday problem solving. Psychol Aging 2007;22:470481.

17 Yaffe K, Lindquist K, Shlipak MG, Simonsick E, Fried L, Rosano C, Satterfield S, Atkinson $H$, Windham BG, Kurella-Tamura M: Cystatin $\mathrm{C}$ as a marker of cognitive function in elders: findings from the health $\mathrm{ABC}$ study. Ann Neurol 2008;63:798-802.
18 Ikram MA, Vernooij MW, Hofman A, Niessen WJ, van der Lugt A, Breteler MM: Kidney function is related to cerebral small vessel disease. Stroke 2008;39:55-61.

19 Seliger SL, Longstreth WT Jr, Katz R, Manolio T, Fried LF, Shlipak M, Stehman-Breen CO, Newman A, Sarnak M, Gillen DL, Bleyer A, Siscovick DS: Cystatin C and subclinical brain infarction. J Am Soc Nephrol 2005; 16:37213727.

20 Seliger SL, Siscovick DS, Stehman-Breen CO, Gillen DL, Fitzpatrick A, Bleyer A, Kuller LH: Moderate renal impairment and risk of dementia among older adults: the cardiovascular health cognition study. J Am Soc Nephrol 2004;15:1904-1911.

21 Markham JA, McKian KP, Stroup TS, Juraska JM: Sexually dimorphic aging of dendritic morphology in CA1 of hippocampus. Hippocampus 2005;15:97-103.

22 Barzilay JI, Fitzpatrick AL, Luchsinger J, Yasar S, Bernick C, Jenny NS, Kuller LH: Albuminuria and dementia in the elderly: a community study. Am J Kidney Dis 2008;52:216226.

23 Weiner DE, Bartolomei K, Scott T, Price LL, Griffith JL, Rosenberg I, Levey AS, Folstein MF, Sarnak MJ: Albuminuria, cognitive functioning, and white matter hyperintensities in homebound elders. Am J Kidney Dis 2009;53: 438-447.

24 Shlipak MG, Katz R, Kestenbaum B, Siscovick D, Fried L, Newman A, Rifkin D, Sarnak MJ: Rapid decline of kidney function increases cardiovascular risk in the elderly. J Am Soc Nephrol 2009;20:2625-2630. 